\title{
Humane Milch-Oligosaccharide in aller (Babys) Munde
}

\author{
Barbara Petschacher
}

\author{
Wissenschaftlern weltweit gelingt es zunehmend, die wertvollen Inhaltsstoffe von Mutter- \\ milch und ihre große Bedeutung für die Säuglingsgesundheit zu entschlüsseln. Das Institut \\ für Biotechnologie und Bioprozesstechnik der TU Graz forscht zum Thema humane Milch- \\ Oligosaccharide (HMO). In ihrem Übersichtsartikel erklärt die Autorin bisherige Erkenntnisse \\ über den Aufbau, das Vorkommen und die Wirkungsweise von HMO. Daraus ergeben sich \\ spannende Perspektiven für mögliche zukünftige Anwendungen.
}

\section{Hintergrund}

Muttermilch ist der von der WHO empfohlene Goldstandard für die Ernährung von Neugeborenen besonders in den ersten 6 Lebensmonaten. Sie enthält alle für ein gesundes Kind notwendigen Nährstoffe wie Kohlenhydrate, Fette und Proteine, Vitamine, Mineralstoffe und Spurenelemente. Darüber hinaus enthält Muttermilch aber auch biologisch aktive Substanzen mit speziellen gesundheitsfördernden Wirkungen für den Säugling. Zu diesen Substanzen gehören auch humane Milch-Oligosaccharide, eine Mischung aus komplexen Mehrfachzuckern, die für den Menschen unverdaulich sind [1].

Mengenmäßig stellt die Fraktion der HMO nach Laktose und Fett mit durchschnittlich 10-15 g/L den drittgrößten Bestandteil von Muttermilch dar [2]. Um ca. 1930 wurde erkannt, dass der sogenannte „bifidogene Effekt“ der Muttermilch, also die Förderung der Vermehrung von nützlichen Bifidobakterien im Darm des Kindes, auf diese Kohlenhydratfraktion zurückzuführen ist. Um 1950 konnten die ersten HMO-Strukturen aufgeklärt werden [3]. Seither wird intensiv an HMO und deren Wirkung geforscht.

Die Milch von Tieren wie Kühen, Schafen oder Ziegen weist im Vergleich zu menschlicher Muttermilch 1001000-mal geringere Konzentrationen an HMO auf, einige wichtige $\mathrm{HMO}$, wie z. B. Fucosyllactose, kommen darin nur in Spuren vor [4][5]. Menschliche Muttermilch ist also für die Ernährung von Babys auch in Hinsicht auf diese speziellen Oligosaccharide von großer Bedeutung.

Heute stehen wir an einem Wendepunkt der HMO-Forschung, da erstmals große Mengen zumindest einiger dieser gesundheitlich wertvollen Zucker hergestellt werden können, ohne dabei auf Muttermilch als Ausgangsstoff angewiesen zu sein. So können nun breit angelegte
Wirkungsstudien mit den häufigsten HMO durchgeführt werden. Diese HMO stehen nun auch großflächig Kindern, die nicht gestillt werden können, in Muttermilchersatznahrung zur Verfügung.

\section{Struktur und Vorkommen von HMO}

An die 200 einzelne Strukturen von HMO konnten bisher identifiziert werden [6]. All diese Varianten an Oligosacchariden werden aus nur fünf Grundbausteinen aufgebaut, den Einfachzuckern Glukose, Galaktose, $\mathrm{N}$-Acetylglucosamin, Sialinsäure (in Form von N-Acetylneuraminsäure) und Fucose. Bei der Bildung der HMO in der Milchdrüse wird das Disaccharid Laktose, bestehend aus Glukose und Galaktose, mithilfe von Enzymen durch weitere Disaccharide aus Galaktose und N-Acetylglucosamin verlängert. Bis zu 15 und mehr solcher Disaccharideinheiten werden dabei auf verschiedene Arten miteinander verknüpft, sodass verzweigte und unverzweigte Mehrfachzucker entstehen. Diese können dann noch endständig mit den Einfachzuckern Fucose und N-Acetylneuraminsäure modifiziert werden [7]. Der Dreifachzucker 2'-Fucosyllactose stellt bei einem Großteil der Frauen mit einem Anteil an allen $\mathrm{HMO}$ von durchschnittlich ca. $30 \%$ das höchstkonzentrierte $\mathrm{HMO}$ dar. Allerdings unterliegt die Konzentration an $\mathrm{HMO}$ in Muttermilch starken Schwankungen. Zum Einen ändert sich der Anteil an HMO über die Stillzeit. Im Kolostrum ist die Konzentration mit 20-25 g/L am höchsten. Über die Laktationsperiode sinkt die Menge dann auf $10-15 \mathrm{~g} / \mathrm{L}[4]$.

\section{Neueste Vergleiche der Daten}

Frühere Ergebnisse aus Forschungen, wonach die Milch frühgebärender Frauen besonders viele HMO enthält, konnten nicht bestätigt werden [8][9]. 
Ein weiterer Faktor, der die Zusammensetzung der HMO in der Muttermilch beeinflusst, ist die genetische Grundausstattung der Mutter. Nicht alle Frauen besitzen alle notwendigen Enzyme um die verschiedenen Zuckerverknüpfungen zu katalysieren. Diese genetische Ausstattung spiegelt sich auch in den sogenannten LewisBlutgruppenmerkmalen der Frauen wider, da dieselben Enzyme auch für die Dekoration der Blutkörperchen mit Zuckerketten zuständig sind. Manche Frauen tragen kein Gen für die Expression des Enzyms FUT-2 (sie werden als Sekretor negativ oder Se- bezeichnet), anderen fehlt das Enzym FUT-3 (Lewis negativ). Wenige Frauen können beide Enzyme nicht bilden.

Sekretor negative Frauen können praktisch keine 2'-Fucosyllactose bilden. Unter Europäerinnen und Amerikanerinnen sind ca. $75 \%$ Sekretor positiv [10][11]. Milch Sekretor negativer Mütter weisen um 35-45\% weniger HMO (darunter hauptsächlich 2'- Fucosyllactose) auf als Milch Sekretor positiver Mütter [10].

Tatsächlich kommen auch manche positiven Effekte des Stillens bei Sekretor negativen Frauen weniger zum Tragen als bei Se+ Müttern. Es konnte gezeigt werden, dass Kinder von Sekretor negativen Müttern, die durch Kaiserschnitt auf die Welt gebracht werden, eine vermehrte Enterokokken-und eine verringerte Bifidobakterienpopulation aufwiesen. Bei Kaiserschnitt-Kindern Sekretorpositiver Mütter, deren Milch also eine höhere Menge an $\mathrm{HMO}$ enthielt, war das Mikrobiom wesentlich ähnlicher dem von natürlich geborenen Babys [12]. Allgemein ist der Einfluss des Stillens auf das Mikrobiom bis ca. 3 Jahre nach der Geburt bemerkbar. Danach stellt sich das Mikrobiom entsprechend den Ernährungs- und Lebensbedingungen ein und lässt keine Rückschlüsse mehr darauf zu, ob ursprünglich Muttermilch oder Muttermilchersatznahrung erhalten wurde [13].

\section{Wirkung der HMO}

Auch wenn der bifidogene Effekt, also die präbiotische Wirkung der HMO, als erste gesundheitsfördernde Wirkung dieser besonderen Zucker erkannt wurde, weiß die Wissenschaft heute von vielen weiteren und komplexeren Wirkungen auf die Gesundheit der Neugeborenen.

\section{Präbiotischer Effekt}

Als Grundvoraussetzung für den präbiotischen Effekt auf das Mikrobiom im Dickdarm müssen HMO Magen und Dünndarm unverdaut passieren. Der Mensch besitzt die zur Spaltung der Mehrfachzucker notwendigen Enzyme nicht. So gelangen HMO in tiefere Darmregionen. Dort tragen sie maßgeblich zur Ausgestaltung des sogenannten Darmmikrobioms, also der Gesamtheit an Bakterien, Archaeen, Pilzen und Viren im Darm bei. Die Darmflora spielt für die Gesundheit des Menschen eine erstaunlich große Rolle.
Eine gesunde Darmflora wirkt auf viele Aspekte menschlicher Gesundheit, vom Metabolismus bis zum Verhalten [14]. Sie trägt zur Ausbildung einer Schutzschicht auf den Darmzellen bei, sie wehrt pathogene Keime ab und versorgt mit ihren Stoffwechselprodukten die Darmzellen mit wichtigen Nährstoffen. Hier kommt den von den Bakterien erzeugten kurzkettigen Fettsäuren eine besondere Bedeutung zu, die einerseits den $\mathrm{pH}$-Wert ins Saure verschieben und damit eine Umgebung erzeugen, die gutartige Keime im Gegensatz zu Pathogenen bevorzugt besiedeln. Andererseits dienen diese kurzkettigen Fettsäuren den Epithelzellen als Nahrung [15].

Für ein gesundheitsförderndes Darmmikrobiom ist die Zusammensetzung der Bakterienpopulation von entscheidender Bedeutung. Ein Missverhältnis der Bakterienstämme im Darm wird mit Krankheiten wie Stoffwechselstörungen, Entzündungen, Diabetes und Zöliakie assoziiert [16]. Die Zusammensetzung des Mikrobioms, die sich am Anfang des Lebens etabliert, wird von vielen Faktoren beeinflusst. Die Art der Geburt, Art und Inhalt der Ernährung, Umwelteinflüsse sowie Antibiotikabehandlungen beeinflussen die Ansiedlung der Mikroorganismen [10]. Hier nun entfalten HMO im noch dünn besiedelten Darm der Neugeborenen ihre volle präbiotische Wirkung. Sie können von bestimmten gesundheitsfördernden Bakterien wie Bifidobacterium longum subs. infantis und anderen Bifidobakterien verstoffwechselt werden [17]. So tragen sie zu deren verstärkter Vermehrung und damit zur Unterdrückung schädlicher Bakterien und zur Ausbildung einer gesunden Darmflora bei.

\section{Antiadhäsiver Effekt}

Neben der wachstumsfördernden Wirkung auf Bakterien mit positivem Effekt auf die Gesundheit sind HMO auch direkt in die Abwehr pathogener Keime involviert. Auch menschliche Darmepithelzellen tragen Zuckerketten an der Oberfläche, die den HMO sehr ähnlich sind. Pathogene Keime nutzen diese Zuckerketten als Andockstellen und ersten Schritt für eine Invasion des Wirtsorganismus. Durch die Muttermilch werden nun in substanziellen Mengen HMO zugeführt, die dieselben Strukturen wie die Zelloberflächenzuckerketten aufweisen. Pathogene Bakterien erkennen die Zuckerstrukturen und binden nun HMO statt der Zuckerketten der Epithelzellen. Da HMO aber in freier Form vorliegen, werden die an HMO gebundenen Pathogene schlussendlich über den Stuhl aus dem Darm entsorgt. Es konnte gezeigt werden, dass die Aufnahme von 2'Fucosyllactose die Bindung von Campylobacter jejuni in Mäusen um $80 \%$ reduzierte und Darmentzündungen verringerte [18]. Campylobacter ist eine der Hauptursachen für Darmentzündungen und Durchfallerkrankungen bei Neugeborenen. Unter anderem können auch Infektionen mit Entamoeba histolytica, Escherichia coli, 
Vibrio cholerae, Noro- und Rotaviren sowie HIV mithilfe von HMO verringert werden [19][20].

Eine besonders wichtige Rolle spielen auch Untersuchungen zum Einfluss von HMO auf nekrotisierende Enterokolitis (NEC), eine entzündliche Darmerkrankung, die besonders für Frühgeborene eine große Gefahr darstellt. 7-14\% der Frühgeborenen werden von NEC befallen, die Sterblichkeit ist hoch. Forscher haben entdeckt, dass speziell das HMO Disialyllacto-N-tetraose (DSLNT) vor NEC schützen kann [21].

\section{Antimikrobielle Wirkung}

Neben ihren präbiotischen und antiadhäsiven Effekten können HMO auch direkt antimikrobiell wirken. So konnte gezeigt werden, dass Streptococcous agalactiae ein Bakterium, das zu Fehlgeburten führen kann, nach Einbau von $\mathrm{HMO}$ in seine Zellmembran nicht mehr vermehrungsfähig ist [7]. HMO scheinen auch einen Pilzbefall durch Candida albicans durch direkte Eindämmung des Hyphenwachstums deutlich zu reduzieren [22].

\section{Modulierung der Epithelzellen, Immunmodulierung}

Eine wachsende Zahl an Studien zeigt, dass HMO auch direkt auf Epithel- und Immunzellen wirken. Sie können die Expression verschiedener Enzyme beeinflussen und so Veränderungen in den Zuckerketten an der Epithelzellenoberfläche bewirken oder die Cytokinproduktion in Lymphozyten beeinflussen. Ungefähr $1 \%$ der mit der Nahrung aufgenommenen HMO werden in Magen und Darm absorbiert, gelangen also ins Blutsystem und können damit auch eine systemische Wirkung an anderen Orten als im Magen-Darmtrakt entfalten. So konnte gezeigt werden, dass HMO auch Epithelzellen des Urogenitaltrakts resistent gegen Angriffe von Escherichia coli Bakterien machen [23]. In Tiermodellen konnten HMO auch die Entwicklung von Diabetes Typ 1 verzögern oder verhindern und die Wirkung von Grippeimpfungen verbessern [24][25]. Neueste Ergebnisse legen nahe, dass bestimmte HMO die Wirksamkeit von Rotavirus Lebendimpfstoffen steigern können. Der durch das Stillen erhaltene HMO Mix nimmt also Einfluss auf die Wirkung der Impfung [26], ein Effekt, der aufzeigt, wie wichtig genaue Wirkstudien für HMO sind.

\section{Hirnentwicklung}

HMO wirken sich auch auf vom Darm weit entfernte Körperregionen wie das Gehirn aus. Einerseits wirken Metabolite des HMO-Abbaus, speziell Sialinsäure, positiv auf die Hirnentwicklung, Nervenleitung und Synapsenbildung [27]. Auch die Lernfähigkeit von Nagetieren konnte durch 2'-Fucosyllactose gesteigert werden [28]. Andererseits bewirkt ein durch $\mathrm{HMO}$ gefördertes gesundes Mikrobiom über eine Verbindung zwischen Darmmikrobiom und Hirn eine erhöhte Stressresistenz [29]. 


\section{Zukünftige Anwendungen}

Die Anwendung von HMO könnte in Zukunft noch weit über den Einsatz in Muttermilchersatznahrung hinausgehen. So konnte gezeigt werden, dass 6 '-Sialyllactose und 2'-Fucosyllactose die Reaktion menschlicher Epithelzellen bei Allergieerkrankungen beeinflussen [30]. Diese Ergebnisse befürworten weitere Untersuchungen des therapeutischen Potenzials von HMO bei Nahrungsmittelallergien.

HMO könnten auch Erwachsenen helfen, ein ausgewogenes Mikrobiom etwa im Fall einer Antibiotikabehandlung oder im Fall einer Darmresektion wiederherzustellen [31][32]. Ihr Einsatz als Nahrungsergänzungsmittel zum Beispiel in Joghurts wird daher überlegt. Die Gabe von Fucosyllactose konnte auch die Wirkung von etlichen Antibiotika verstärken [33]. HMO könnten zukünftig also auch eine Rolle in der Bekämpfung aufkommender Antibiotikaresistenzprobleme spielen.

\section{Bedeutung für Babynahrung}

Für Kinder, die nicht gestillt werden können, ist die Verfügbarkeit von Babynahrung, die der Muttermilch so ähnlich wie möglich ist, essenziell. Da humane Milch-Oligosaccharide bis vor kurzem nicht in großen Mengen hergestellt werden konnten, wurde die präbiotische Wirkung von Muttermilch durch den Einsatz anderer pflanzenbasierter Oligosaccharide nachempfunden. Dabei kommen vorrangig Fructooligosaccharide (FOS) und Galactooligosaccharide (GOS) zum Einsatz, die präbiotische Effekte für viele Mikroorganismen aufweisen [34]. Spezifische Wirkungen, die von der exakten Struktur der HMO abhängen, können durch GOS und FOS aber nicht ersetzt werden. So können Enterobacteriaceae, unter ihnen auch pathogene Stämme, in vitro auf GOS wachsen, während getestete HMO keinen Nährstoff für die Vermehrung dieser Bakterien bieten [35].

In den letzten Jahren wurde die fermentative Herstellung von den einfachsten $\mathrm{HMO}$, wie 2'-Fucosyllactose und Lacto-N-neotetraose (LNnT) vom Labormaßstab in den industriellen Maßstab weiterentwickelt [36], sodass nun HMO im Multitonnenmaßstab hergestellt werden können. Besonders wichtig dabei ist, dass die Herstellungsprozesse der synthetischen $\mathrm{HMO}$, die chemisch ident mit den in Muttermilch enthaltenen sind, die strengen regulatorischen Zulassungsverfahren in Europa und den USA passiert haben.

Es konnte in Studien gezeigt werden, dass Muttermilchersatznahrung, die mit 2'-Fucosyllactose und LNnT supplementiert ist, keine negative Wirkung auf Säuglinge hat [37]. Säuglinge, die GOS in Kombination mit Fucosyllactose erhielten, zeigten auch eine dem gestillter Kinder ähnlichere Immunsystementwicklung im Vergleich zu Kindern, die nur GOS erhielten [38]. Die erste klinische Studie über mit HMO supplementierte Muttermilchersatznahrung zeigte positive Effekte wie geringeres Aufwachen in der Nacht und weniger Koliken bei Kaiserschnittkindern. Eltern berichteten über weniger Bronchitis und andere Infekte der Atemwege sowie einen zweifach geringeren Bedarf an Antibiotika. Der Stuhl der Kinder war signifikant weicher. Das Mikrobiom der Kinder, die HMO erhielten, war dem gestillter Kinder ähnlicher [39].

\section{FAZIT}

\section{Wissen für die Wochenbettbetreuung}

Humane Milch-Oligosaccharide sind eine wichtige gesundheitsfördernde Kohlenhydratfraktion in menschlicher Muttermilch.

HMO wirken präbiotisch, antiadhäsiv und antimikrobiell. Sie können Epithelzellen des Darms und die Immunabwehr modulieren und sind für die Hirnentwicklung wichtig.

Bisher wurden alternativ zu HMO der Muttermilchersatznahrung FOS und GOS als Präbiotika beigefügt, die aber nicht alle Wirkungen der HMO erzielen können.

Seit kurzem können die einfachsten HMO industriell hergestellt werden. Synthetische HMO sind bereits in Amerika und Europa für den Einsatz in Muttermilchersatznahrung zugelassen.

Derzeit sind noch wenige Studien zum Einsatz von $\mathrm{HMO}$ in Kindernahrung verfügbar, erste Ergebnisse sind vielversprechend. Es bedarf noch einiger weiterer Zeit und weiterer unabhängiger Untersuchungen, um zu klären, wie weit sich die vielversprechenden Forschungsergebnisse zu positiven Wirkungen der HMO auch im täglichen Einsatz in Muttermilchersatznahrung zeigen. Durch die nun gewährleistete Verfügbarkeit an HMO ist in nächster Zeit mit einem großen Anstieg an Erfahrungen mit HMO-Supplementierung zu rechnen. Die Industrie arbeitet daran, weitere $\mathrm{HMO}$ -

Varianten, insbesondere auch sialylierte Formen, zur Verfügung zu stellen. Eine Ausweitung von derzeit zwei auf zumindest fünf verfügbare Strukturen ist geplant, um die wertvollen Eigenschaften der Muttermilch möglichst naturgetreu nachbilden zu können.

Darüber hinaus warten auch neue Einsatzmöglichkeiten für Erwachsene als Nahrungsergänzungsmittel oder im therapeutischen Bereich auf ihre Erforschung. 


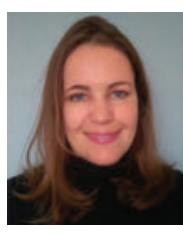

Dipl.-Ing. Dr. techn. Barbara Petschacher forscht am Institut für Biotechnologie und Bioprozesstechnik der Technischen Universität Graz und im Rahmen des ACIB - Austrian Centre of Industrial Biotechnology in der Arbeitsgruppe von Univ.-Prof. Bernd Nidetzky an Enzymen für die biotechnologische Produktion von $\mathrm{HMO}$

\section{Korrespondenzadresse}

Dr. Barbara Petschacher

Institut für Biotechnologie und Bioprozesstechnik

Technische Universität Graz

NAWI Graz

Petersgasse $12 / 1$

$8010 \mathrm{Graz}$

Österreich

E-Mail: barbara.petschacher@tugraz.at

\section{Literatur}

[1] Bode L. Human milk oligosaccharides: Every baby needs a sugar mama. Glycobiology 2012; 22: 1147-1162
[2] Zivkovic A, German J, Lebrilla C et al. Human milk glycobiome and its impact on the infant gastrointestinal microbiota. Proc Natl Acad Sci U S A 2011, 108, 4653-4658

[3] Kuhn R, Baer H. Die Konstitution der Lacto-N-tetraose. Chem Ber 1956; 89: 504-511

[4] Urashima T, Taufik E, Fukuda $\mathrm{K}$ et al. Recent advances in studies on milk oligosaccharides of cows and other domestic farm animals. Biosci Biotechnol Biochem 2013; 77: 455-466

[5] Albrecht S, Lane J, Mariño $\mathrm{K}$ et al. A comparative study of free oligosaccharides in the milk of domestic animals. $\mathrm{Br}$ J Nutr 2014; 111: 1313-1328

[6] Ninonuevo M, Park $\mathrm{Y}$, Yin $\mathrm{H}$ et al. A strategy for annotating the human milk glycome, J Agric Food Chem 2006, 54: 74717480

[7] Bode L. The functional biology of human milk oligosaccharides. Early Hum Dev 2015; 91: 619-622

[8] Kunz C, Meyer C, Collado M et al. Influence of gestational age, secretor, and lewis blood group status on the oligosaccharide content of human milk. J Pediatr Gastroenterol Nutr 2017; 64: 789-798

[9] Thurl S, Munzert M, Boehm G et al. Systematic review of the concentrations of oligosaccharides in human milk. Nutr Rev 2017; 75: 920-933

[10] Azad M, Robertson B, Atakora F et al. Human milk oligosaccharide concentrations are associated with multiple fixed and modifiable maternal characteristics, environmental factors, and feeding practices. I Nutr 2018; 148: 1733-1742 
[11] McGuire M, Meehan C, McGuire M et al. What's normal? Oligosaccharide concentrations and profiles in milk produced by healthy women vary geographically. Am J Clin Nutr 2017; 105: 1086-1100

[12] Korpela K, Salonen A, Hickman B et al. Fucosylated oligosaccharides in mother's milk alleviate the effects of caesarean birth on infant gut microbiota. Sci Rep 2018; 8: 13757

[13] Bergström A, Skov T, Bahl M et al. Establishment of intestinal microbiota during early life: a longitudinal, explorative study of a large cohort of Danish infants. Appl Environ Microbiol 2014; 80: 2889-2900

[14] Lloyd-Price J, Abu-Ali G, Huttenhower C. The healthy human microbiome. Genome Med 2016; 8: 51

[15] Figueroa-Lozano S, de Vos P. Relationship between oligosaccharides and glycoconjugates content in human milk and the development of the gut barrier. Compr Rev Food Sci Food Saf 2018, early view

[16] Kho ZY, Lal SK. The human gut microbiome-A potential controller of wellness and disease. Front Microbiol 2018; 9: 1835

[17] Thomson P, Medina D, Garrido D. Human milk oligosaccharides and infant gut bifidobacteria: Molecular strategies for their utilization. Food Microbiol 2018; 75: 37-46

[18] Yu Z-T, Nanthakumar N, Newburg D. The human milk oligosaccharide 2'-fucosyllactose quenches Campylobacter jejuniinduced inflammation in human epithelial cells HEp-2 and HT-29 and in mouse intestinal mucosa. J Nutr 2016; 146: 1980-1990

[19] Coppa G V, Zampini L, Galeazzi T et al. Human milk oligosaccharides inhibit the adhesion to Caco-2 cells of diarrheal pathogens: Escherichia coli, Vibrio cholerae, and Salmonella fyris. Pediatr Res 2006; 59: 377-382

[20] Morozov V, Hansman G, Hanisch F-G et al. Human milk oligosaccharides as promising antivirals. Mol Nutr Food Res 2018; 62: 1700679

[21] Jantscher-Krenn E, Zherebtsov M, Nissan C et al. The human milk oligosaccharide disialyllacto-N-tetraose prevents necrotising enterocolitis in neonatal rats. 2012; 61: 1417-1425

[22] Gonia S, Tuepker M, Heisel T et al. Human milk oligosaccharides inhibit Candida albicans invasion of human premature intestinal epithelial cells. J Nutr 2015; 145: 1992-1998

[23] Lin A, Autran C, Espanola S, et al. Human milk oligosaccharides protect bladder epithelial cells against uropathogenic Escherichia coli invasion and cytotoxicity. J Infect Dis 2014; 209: 389-398

[24] Xiao L, van't Land B, Engen P et al. Human milk oligosaccharides protect against the development of autoimmune diabetes in NOD-mice. Sci Rep 2018; 8: 3829

[25] Xiao L, Leusink-Muis T, Kettelarij $\mathrm{N}$ et al. Human milk oligosaccharide 2'-Fucosyllactose improves innate and adaptive immunity in an influenza-specific murine vaccination model. Front Immunol 2018; 9: 452

[26] Ramani S, Stewart C, Laucirica D, et al. Human milk oligosaccharides, milk microbiome and infant gut microbiome modulate neonatal rotavirus infection. Nat Commun 2018; 9: 5010
[27] Vandenplas Y, Berger B, Carnielli V et al. Human milk oligosaccharides: 2'-fucosyllactose (2'-FL) and lacto-n-neotetraose (LNnT) in infant formula. Nutrients 2018; 10: 1161

[28] Vázquez E, Barranco A, Ramírez M, et al. Effects of a human milk oligosaccharide, 2'-fucosyllactose, on hippocampal longterm potentiation and learning capabilities in rodents. J Nutr Biochem 2015; 26: 455-465

[29] Tarr A, Galley J, Fisher S et al. The prebiotics 3'Sialyllactose and 6'Sialyllactose diminish stressor-induced anxiety-like behavior and colonic microbiota alterations: Evidence for effects on the gut-brain axis. Brain Behav Immun 2015; 50: 166-177

[30] Zehra S, Khambati I, Vierhout $\mathrm{M}$ et al. Human milk oligosaccharides attenuate antigen-antibody complex induced chemokine release from human intestinal epithelial cell lines. J Food Sci 2018; 83: 499-508

[31] Elison E, Vigsnaes L, Rindom Krogsgaard L et al. Oral supplementation of healthy adults with 2'-O-fucosyllactose and lacto- $\mathrm{N}$-neotetraose is well tolerated and shifts the intestinal microbiota. Br J Nutr 2016; 116: 1356-1368

[32] Mezoff E, Hawkins J, Ollberding $\mathrm{N}$ et al. The human milk oligosaccharide 2'-fucosyllactose augments the adaptive response to extensive intestinal. Am J Physiol - Gastrointest Liver Physiol 2016; 310: G427-G438

[33] Craft K, Gaddy J, Townsend S. Human Milk Oligosaccharides (HMOs) sensitize group B Streptococcus to Clindamycin, Erythromycin, Gentamicin, and Minocycline on a strain specific basis. ACS Chem Biol 2018; 13: 2020-2026

[34] G. Moro, I Minoli, Mosca M et al. Dosage-related bifidogenic effects of galacto- and fructooligosaccharides in formula-fed term infants. J Pediatr Gastroenterol Nutr 2002; 34: 291-295

[35] Hoeflinger J, Davis S, Chow J et al. In vitro impact of human milk oligosaccharides on Enterobacteriaceae growth. J Agric Food Chem 2015; 63: 3295-3302

[36] Bych K, Mikš M, Johanson T et al. Production of HMOs using microbial hosts - from cell engineering to large scale production. Curr Opin Biotechnol 2019; 56: 130-137

[37] Marriage B, Buck R, Goehring K et al. Infants fed a lower calorie formula with 2'FL show growth and 2'FL uptake like breast-fed infants. J Pediatr Gastroenterol Nutr 2015; 61: 649-658

[38] Goehring K, Marriage B, Oliver J et al. Similar to those who are breastfed, infants fed a formula containing 2'-fucosyllactose have lower inflammatory cytokines in a randomized controlled trial. J Nutr 2016; 146: 2559-2566

[39] Puccio G, Alliet P, Cajozzo C et al. Effects of Infant Formula With Human Milk Oligosaccharides on Growth and Morbidity. J Pediatr Gastroenterol Nutr 2017; 64: 624-631

\section{Bibliografie}

DOI https://doi.org/10.1055/a-0792-0676

Die Hebamme 2018; 31: 409-414

(c) Georg Thieme Verlag KG Stuttgart · New York

ISSN 0932-8122 